\title{
UV AND RADIOFREQUENCY OBSERVATIONS OF WOLF-RAYET STARS
}

\author{
HUGH M. JOHNSON \\ Lockheed Missiles and Space Company, Palo Alto, Calif., U.S.A.
}

\section{UV Observations with OAO-2}

Very few observations are available. Of early rocket work on $\gamma$ Vel (Stecher and Milligan, 1962; Smith, 1967) the most detailed spectra are by Stecher (1968) in the range $\lambda 1200-3100 \AA$ and by Carruthers (1968) in the range $\lambda 1050-1216 \AA$. West's (1971) spectrum of $\gamma$ Vel with the OAO-2 spectrometer (Code et al., 1970) in the range $\lambda$ 1050-2000 $\AA$ appears to be somewhat different from Stecher's. Comparison must await full details.

The OAO-2 spectrometer has scanned apparently only one other W star, HD 50896. It is more informative than $\gamma$ Vel because it is possibly a single star without contamination by a dominant O-star spectrum. It is also one of the WN5 stars in a symmetric nebula (cf. Section 2). Smith (1971) has reported the OAO-2 spectrum of HD 50896. Let us make a few more remarks about the same scans. First, the measured FWHM of the strong He II 1640 emission is $\Delta \lambda=20 \AA$, or about $17 \AA$ after correction for instrumental broadening, in agreement with the ratio $\Delta \lambda / \lambda$ of fairly unblended lines at wavelengths several times larger. Thus Doppler broadening is confirmed. Second, interstellar hydrogen absorption at $\lambda 1215 \AA$ may be estimated from the $21-\mathrm{cm}$ survey of McGee and Murray (1961): $N_{\mathrm{H}}=2.0 \times 10^{21} \mathrm{H}$ atoms cm $\mathrm{cm}^{-2}$ toward HD 50896. Since the star is at $l=234: 8, b=-10.1$, and at the distance $1.59 \mathrm{kpc}$ (Smith, 1968b),

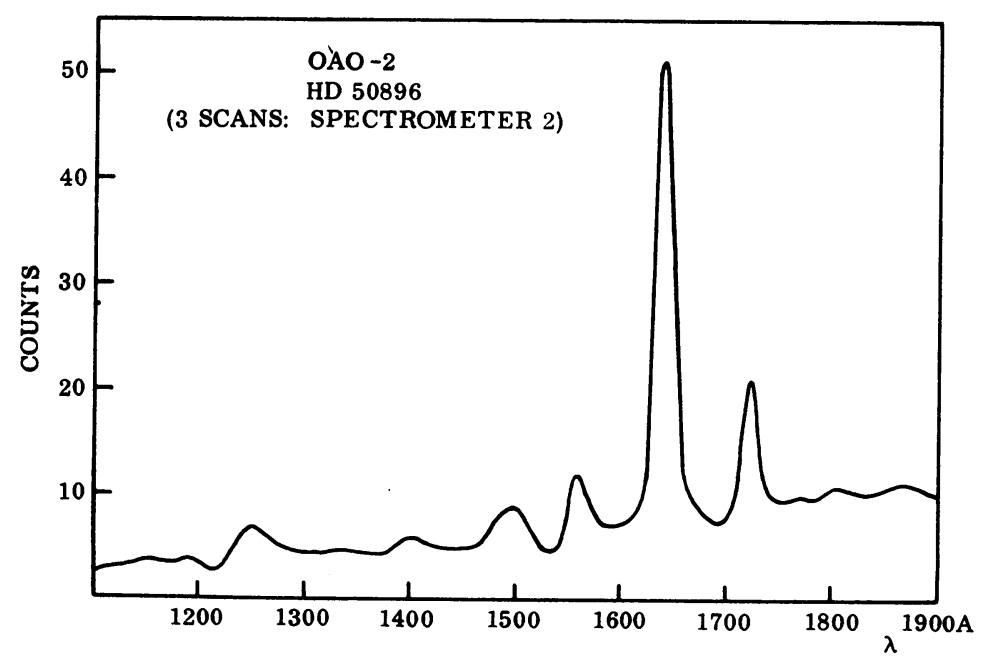

Fig. 1. Scanned spectrum of the WN5 star HD 50896. 
most of the hydrogen should be in front of it. Morton's (1967) formula for the equivalent width $\mathrm{EW}(\mathrm{L} \alpha)=7.31 \times 10^{-10} N_{\mathrm{H}}^{1 / 2}(\mathrm{~A})$ due to radiation damping of the interstellar line, then gives $E W(L \alpha) \leqslant 33 \AA$ in absorption. As estimated from Figure 1 the net equivalent width of the features around $L \alpha$ is $\leqslant 30 \AA$ in emission, so the net equivalent width of the features before interstellar absorption should be $<60 \AA$ in emission. Presumably the dominant emission near $\lambda 1215 \AA$ would be He II 1215 , the second line in the series which He II 1640 heads. We estimate $\operatorname{EW}(\lambda 1640)=140 \AA$, a strength which appears to confirm the absence of continuum from any hot companion star.

The OAO-2 photometers (Code et al., 1970) have been used in the OAO-2 guestinvestigator program to observe HD 192163. This WN 6 star was selected because it is apparently a single star and it is in a symmetric nebula, NGC 6888 (cf. Section 2). In respect to the latter it may not be normal. The four photometers are each equipped with three filters. The range of effective wavelengths $\lambda \lambda 1330-3320 \AA$ is covered with passbands of FWHM $=240-860 \AA$ such that practically no gaps are left in the observed continuum. However, the data will tell little about a line spectrum. Figure 2 shows the observations. Figure 3 corrects them for interstellar extinction by means of the color excess $E_{b-c}=0.33$ mag. for $H D 192163$, and the ratio $E(B-V)=1.6 E_{b-c}$ (Smith and $\mathrm{Kuhi}, 1970)$, and the average ultraviolet extinction $E(\lambda-V) / E(B-V)$ given by Bless and Savage (1971). There is still a question about the calibration of some of the photometer-filter responses (Bless, 1971), but it is apparent that the run of the data corrected according to this differential extinction agrees rather well with an interpolated model atmosphere of $\mathrm{T}_{\text {eff }}=30700 \mathrm{~K}$. This is the effective temperature for HD 192163 which Morton (1970) derived in a completely different way.

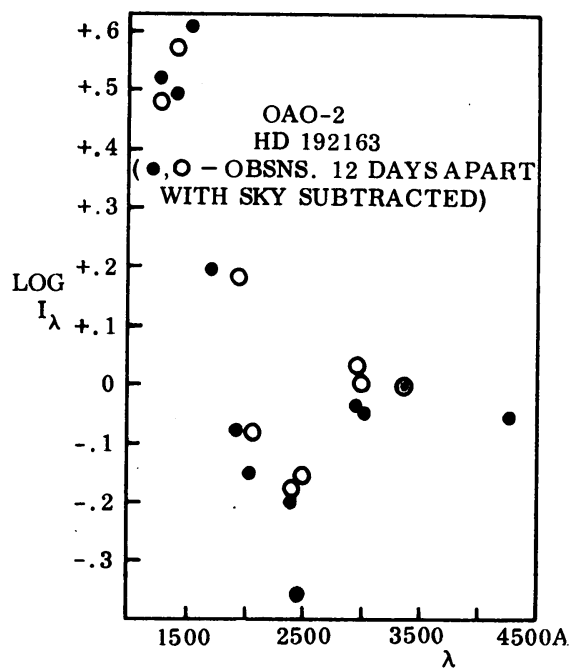

Fig. 2. Photometered spectrum of the WN6 star HD 192163, with intensity per unit wavelength $I_{\lambda}$ normalized to an arbitrary zero-point at $\lambda 3320 \AA$. The first set of data has 12 solid points; the second has 9 open circles. 
Ultraviolet spectral lines might have some effect on Figures 2 and 3, for example the strong line of $\operatorname{EW}(\lambda 1640)=140 \AA$ such as HD 50896 exhibits. It would fall $40 \AA$ off center in the passband with $1 / \lambda_{\text {eff }}=5.95 \mu^{-1}$ and FWHM $=270 \AA$. It should therefore add close to $140 / 270 \times 100 \%=52 \%$ to the light of the continuum, but in fact this point falls low in the plots. We must conclude that $\mathrm{He}$ II 1640 is not prominent in HD 192163. C IV 1548-50 and $\mathrm{N} v 1238-42$ fall in passbands with relatively high points in Figure 3, but we cannot claim the presence of these lines from the data.

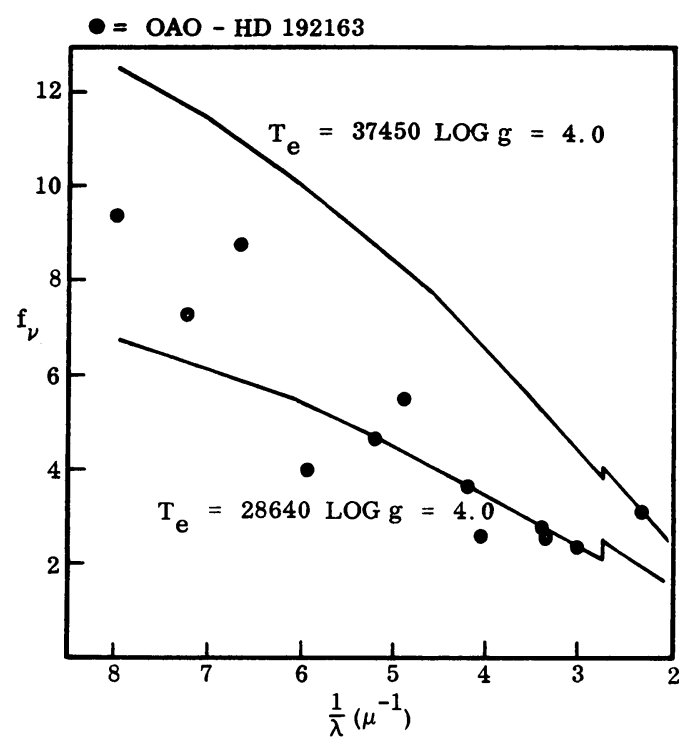

Fig. 3. Mean data of HD 192163 (points) in Figure 2 reduced to intensity per unit frequency, corrected for interstellar extinction, and compared with two model O-type atmospheres (labeled curves) by Bradley and Morton (1969). Dr Morton suggested the comparison with these models. The zero-point of observed intensity is fitted arbitrarily to the scale $f_{v}$ of the curves.

Houck (1971) has made 200 OAO-2 spectrometer scans of $\beta$ Lyr at $10 \AA$ resolution in the range $\lambda \lambda 1100-1800 \AA$. He has found emissions of $C$ IV, Si IV, and ' $L \alpha$ ' (the latter periodically shifted from interstellar absorption by orbital motion) in a spectrum which compares with a star such as $\gamma$ Vel.

Davis (1971) has presented preliminary photometry of about 500 stars observed with the Celescope on OAO-2. Two of them are W stars, CD - $45^{\circ} 4482$ and HD 76536. The new data are magnitudes called $U 1$ and $U 2$, respectively taken with passbands of 2100-3200 $\AA$ and 1550-3200 $\AA$. Despite the gross passbands, additional information on effective temperatures of a few W stars should come out of the final catalog.

\section{Observations of W Stars With Symmetrical Nebulae Around Them}

Johnson and Hogg (1965) used the Green Bank telescopes to detect NGC 6888 around HD 192163 at $750 \mathrm{MHz}$ and $1400 \mathrm{MHz}$; also NGC 2359 around HD 56925 at 750, 
1400 , and $3000 \mathrm{MHz}$. The $\mathrm{W}$ stars are included with the nebulae in the available telescope beams but it has been assumed that the nebulae account for the observed flux densities. Johnson and Hogg (1965) reported the privately communicated independent discoveries by Herbig and by Minkowski of a third nebula $35^{\prime}$ in diameter around HD 50896, which may be called S 308, but it was not detected in radiofrequency until later (Johnson, 1971). Smith $(1967 ; 1968$ a) searched Palomar Sky Survey charts and other material, discovered four more symmetric nebulae around $\mathrm{W}$ stars, and noted that all seven stars were WN 5, 6, or 8 types. Smith and Batchelor (1970) proceeded to observe three of them, NGC 3199, RCW 104, and RCW 58 at $11 \mathrm{~cm}$. Lozinskaya (1970), Terzian (1970), and Johnson (1971) observed NGC 6888 again at 8500,318 , and $7795 \mathrm{MHz}$, respectively. The radiofrequency spectrum of NGC 6888 is

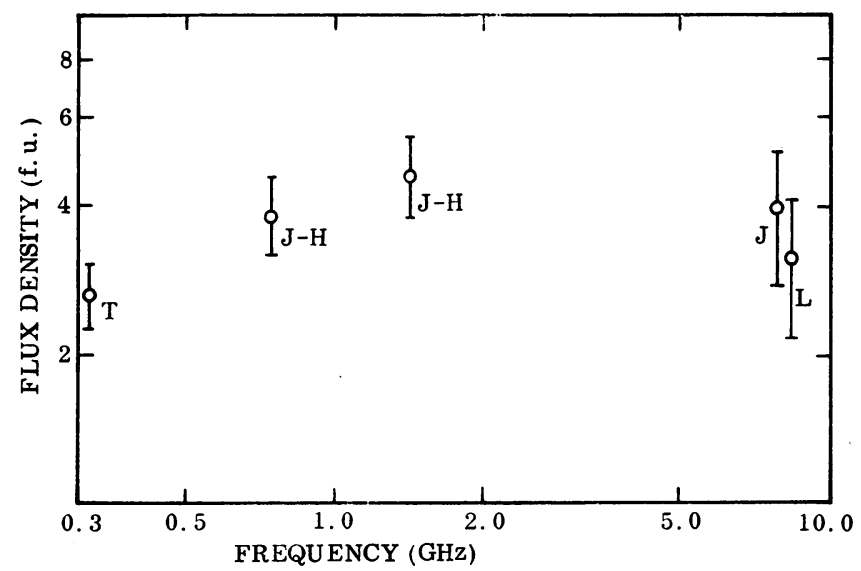

Fig. 4. Radiofrequency spectrum of NGC 6888. Unit flux density (f.u.) $=10^{-26} \mathrm{Wm}^{-2} \mathrm{~Hz}^{-1}$. The observed data are by Johnson and Hogg (1965) J-H, Lozinskay (1970) L, Terzian (1970) T, and Johnson (1971) J.

shown in Figure 4. It is apparently thermal Bremsstrahlung. These radiofrequency data, or alternatively the integrated flux of the nebula in a Balmer line, corrected for extinction, such as Parker (1963) has estimated only for NGC 6888, with a distance estimate of the W star, lead to the mass of the nebula and to the 'excitation parameter' $U$ of the star, or to the equivalent spectral type and effective temperature. The 'Zanstra temperature' of $\mathrm{W}$ stars is also obtained if the stellar magnitude, rather than the distance, is employed with the nebular flux density. Morton (1970) refined the Zanstra method by calculating model atmospheres in place of blackbodies, and he found effective temperatures as a function of W-star types. By including the observed angular size we also get the linear dimensions and a confirmation of the mass distribution (thin-wall shell appearance on photographs). Finally, observations of the internal motions of the nebula may be combined with observations of the rate of mass loss and ejection velocity from the $\mathrm{W}$ star, and with an estimate of ambient interstellar density, in order to obtain the age of the system. 
Conservation of momentum and continuous ejection were assumed in the simple theory (Johnson and Hogg, 1965). Spherical symmetry was also assumed, tacitly contrary to the observed ellipticity of the nebulae, eccentricity of stellar site, and correlated azimuthal asymmetry of nebular perimeter (brightest nearest the star). In apology one can only say that these nebulae are quite symmetrical in comparison with ordinary diffuse nebulae, and perturbations that are attributable to a stellar or interstellar magnetic field, or to irregularity of ambient interstellar density, appear to be negligible in the first objectives of the theory. The higher degree of central-star concentricity which prevails in planetary nebulae may be explained by their smaller radii, statistically higher $z$-distances, and consequent lack of interaction with the ambient medium. However, the non-circular projection of many planetary nebulae shows that central stars do not eject mass isotropically.

The full application of these ideas has been made only to NGC 6888, the W-star nebula in which large nebular velocities are observed (Courtès, 1960); Lozinskaya and Esipov, 1968; Lozinskaya, 1970; Georgelin and Monnet, 1970). The result is a selfconsistent picture of a single WN 6 star with the effective temperature of about $31000 \mathrm{~K}$, which for unknown reasons ejects mass at a velocity of $1400 \mathrm{~km} \mathrm{~s}^{-1}$ and rate of $10^{-5}-10^{-6} \mathrm{M}_{\odot} / \mathrm{yr}$ into collision with an ellipsoidal nebular shell of projected dimensions $12^{\prime} \times 18^{\prime}$. If the distance is $1.2 \mathrm{kpc}$ the mean radius of the shell is $2.6 \mathrm{pc}$, which is considerably larger than the shells of planetary nebulae. Smith (1968b) finds the distance to be $2.29 \mathrm{kpc}$, but this summary is based on the smaller distance. The mean linear thickness of the nebula is only $10^{-2} \mathrm{pc}$ (shell walls); its electron density is $400 \mathrm{~cm}^{-3}$ and electron temperature is $15-19 \times 10^{3} \mathrm{~K}$. The shell is expanding $50-80 \mathrm{~km}$ $\mathrm{s}^{-1}$ and sweeping up interstellar matter with a density of about $1-2 \mathrm{~cm}^{-3}$. The age is about $2 \times 10^{4}$ yrs if the ejection and transfer of momentum has been steady. The shell mass is about $4 \mathrm{M}_{\odot}$ of which only $3 \%$ has been contributed by the ejecta of the W star. Thus the element abundances inferred from the stellar spectrum of HD 192163 need not agree with those inferred from the nebular spectrum (normal). The excitation of the shell appears to be radiative, not collisional.

Before these investigations NGC 6888 was classified as a supernova remnant (e.g. Pikelner, 1959; Lozinskaya and Esipov, 1968) or as a 'giant planetary nebula' (e.g. Parker, 1964). Minkowski (1965) has suggested also that S 308, the shell around HD 50896 , is possibly a planetary nebula. Lozinskaya and Esipov (1968) and Georgelin and Monnet (1970) agree that the mean radial velocity of NGC 6888 is $-50 \mathrm{~km} \mathrm{~s}^{-1}$; no rms error is given. (Lozinskaya and Esipov also estimated the radial velocity of HD 192163 equal to $-120 \pm 20 \mathrm{~km} \mathrm{~s}^{-1}$, despite the difficulty of the broad spectral lines.) The galactic coordinates are $1=75.5, \mathrm{~b}=+2: 4$, the component of differential galactic rotation is $+7 \mathrm{~km} \mathrm{~s}^{-1} \mathrm{kpc}^{-1}$, and the component of solar motion with respect to the local standard of rest is $18 \mathrm{~km} \mathrm{~s}^{-1}$ toward HD 192163 . Hence one component of the velocity of the nebula with respect to its local standard of rest is probably -41 to $-49 \mathrm{~km} \mathrm{~s}^{-1}$ at distances of 1.2-2.3 kpc. Of course, the near side of the nebula may contain most of the observed filaments. If not so, the mean velocity is appropriate to a 'runaway' $O$ star or a planetary nebula. 
In view of the high dispersion of velocities in NGC 6888, it is interesting that three other examples do not show it. They are given in Table I and their velocity dispersions do not differ significantly from those in ordinary nebulae. Successive columns give the nebula, its radius in arc minutes and in pc according to the spectroscopic distance, its mass (Smith and Batchelor, 1970), the rms $\sigma$ of $n$ velocities in the nebula (Georgelin and Georgelin, 1970), the included star, its spectral type, its spectroscopic distance $R(\mathrm{sp})$ (Smith, 1968b), and the kinematical distance of the nebula $R(\mathrm{kin})$ (Georgelin, 1970). If the theory which has been applied usefully to NGC 6888 is applied to the objects of Table I, one must conclude that these nebulae have become massive and the velocities of the shells have slowed down to ordinary internal motions in nebulae,

TABLE I

Symmetric nebulae with small dispersions of velocity

\begin{tabular}{|c|c|c|c|c|c|c|c|c|c|}
\hline \multirow[t]{2}{*}{ Nebula } & \multicolumn{2}{|c|}{ Radius } & \multirow{2}{*}{$\begin{array}{l}\mathbf{M} \\
(\odot)\end{array}$} & \multirow{2}{*}{$\begin{array}{l}\sigma \\
\left(\mathrm{km} \mathrm{s}^{-1}\right)\end{array}$} & \multirow[t]{2}{*}{$n$} & \multirow[t]{2}{*}{ Star } & \multirow[t]{2}{*}{ Sp. } & \multirow{2}{*}{$\begin{array}{l}R(\mathrm{sp}) \\
(\mathrm{kpc})\end{array}$} & \multirow{2}{*}{$\begin{array}{l}R \text { (kin) } \\
(\mathrm{kpc})\end{array}$} \\
\hline & $\overline{\left({ }^{\prime} \text { arc }\right)}$ & (pc) & & & & & & & \\
\hline NGC 2359 & $2^{\mathrm{a}}$ & $4^{a}$ & $330^{a}$ & 8.0 & 29 & HD 56925 & WN5 & 6.92 & 4.57 \\
\hline NGC 3199 & 8 & 8 & 400 & 6.5 & 33 & HD 89358 & WN5 & 3.63 & b \\
\hline RCW $104+106$ & 10 & 18 & 650 & $8.0^{\mathrm{c}}$ & $60^{\mathrm{c}}$ & HD 147419 & WN6 & 6.31 & 3.75 \\
\hline
\end{tabular}

a Pertains to the sharp inner ring of the nebula.

b Differential galactic rotation at $1=283.5$ is too small for a significant kinematical solution.

c Data for both nebulae.

because they are older than NGC 6888. For conventional values of the parameters, e.g. rate of mass loss $=10^{-5} \mathrm{M}_{\odot} / \mathrm{yr}$, velocity of ejection $=10^{3} \mathrm{~km} \mathrm{~s}^{-1}$, and interstellar density $=1$ hydrogen atom $\mathrm{cm}^{-3}$, the derived ages are $2-20 \times 10^{5} \mathrm{yrs}$, or $10-100 \times$ the age of NGC 6888. The difficulty is that WN 6 stars apparently survive $2 \times 10^{6} \mathrm{yrs}$ if HD 192163 and HD 147419 are equal members of the class WN 6. But the total mass loss is $20 \mathrm{M}_{\odot}$ at this age, and the mass of WN 5-6 stars in binary systems is about $11 \mathrm{M}_{\odot}$ (Smith, 1968a). A reduction in rate of mass loss by a factor of 10 increases age by about $\sqrt{ } 10$ so that the total mass loss is then $6 \mathrm{M}_{\odot}$ rather than $20 \mathrm{M}_{\odot}$. An increase of ambient density outside the shell by a factor of 10 also increases age by about $\sqrt{ } 10$.

The mass of RCW 58 is estimated to be small, 5.7 $\mathrm{M}_{\odot}$ (Smith and Batchelor, 1970), and likewise the mass of $\mathrm{S} 308$ to be only $3 \mathrm{M}_{\odot}$ (Johnson, 1971). They are candidates for large expansion velocities but they have not been observed with the interferometer. Neither has any symmetric nebula around a $W$ star been reported in the radiofrequency $\mathbf{H}$ recombination lines. It is reasonable to consider measuring proper-motion expansions in NGC 6888 and S 308, but RCW 58 may be too small for the scale of available plates. At present we tentatively conclude that the less massive nebulae of the class we have discussed are the younger, and that the best-studied member of the class, NGC 6888 , may be peculiar as well as young.

If we accept the theory of ejected stellar mass sweeping out a volume of ambient interstellar gas around some W stars, and note the result that the star contributes only a few per cent of the total mass in each case, we may derive the ambient interstellar 
density $N_{\mathrm{H}}$ of the swept volume. This is, first, a check on self-consistency of the theory for which Johnson and Hogg (1965) originally had to estimate $N_{\mathrm{H}}$ independently in order to establish the theory; and, second, it makes possible some comparisons with the rival suggestion that the gas in a symmetric nebula has been ejected from the star at some earlier stage of evolution(e.g. red giant) and said gas might have no dependence on current mass-loss in the W star. The four nebulae of Table II are the only ones for which the symmetric-nebular mass $\mathbf{M}$ has been derived from the spectroscopic distance $R$, the observed flux density, and the observed nebular gas density $N_{e}$ via the method of the [O II] 3726-29 doublet-ratio. If the gas is non-uniform, $N_{e}$ is overestimated and $\mathrm{M}$ and $N_{\mathrm{H}}$ are underestimated. We draw the following conclusions from the table: first, there is an apparent dependence of $N_{\mathbf{H}}$ on the $z$-distance from the galactic plane,

TABLE II

Interstellar densities near W stars

\begin{tabular}{lllllllll}
\hline Nebula & Star & Sp. & $\begin{array}{l}R(\mathrm{sp}) \\
(\mathrm{kpc})\end{array}$ & $\begin{array}{l}z \\
(\mathrm{pc})\end{array}$ & $\begin{array}{l}r \\
(\mathrm{pc})\end{array}$ & $\begin{array}{l}N_{e}(\mathrm{sp}) \\
\left(\mathrm{cm}^{-3}\right)\end{array}$ & $\begin{array}{l}\text { M } \\
(\odot)\end{array}$ & $\begin{array}{l}N_{H} \\
\left(\mathrm{~cm}^{-3}\right)\end{array}$ \\
\hline NGC 2359 & HD 56925 & WN5 & 6.92 & 12 & $4^{\mathrm{a}}$ & $100^{\mathrm{a}}$ & $330^{\mathrm{a}}$ & \multicolumn{5}{|c}{50} \\
NGC 3199 & HD 89358 & WN5 & 3.63 & 63 & 8 & 240 & 400 & 5.7 \\
NGC 6888 & HD 192163 & WN6 & 2.29 & 96 & 5 & 400 & 21 & 1.2 \\
RCW 104 & HD 147419 & WN6 & 6.31 & 165 & 18 & 190 & 650 & 0.8 \\
\hline
\end{tabular}

a Pertains to the sharp inner ring of the nebula.

in the sense expected of swept interstellar gas rather than of a mass entirely derived from the star during stellar evolution. Second, $N_{\mathrm{H}}$ is a factor of 10 greater than mean interstellar densities, so that an association of $\mathrm{W}$ stars with denser clouds is implied. Alternatively, we could say that $N_{\mathrm{H}}$ decreases from type WN 5 to type WN 6, and the absolute density is governed by factors other than ambient interstellar mean density to make it greater than the mean. At present the interstellar density near specific W stars is not well enough known independently to decide.

It is interesting to ask about the visibility of the ejecta of $\mathrm{W}$ stars before any effects of collision with ambient interstellar matter. If the velocity of the mass lost at radii $r \geqslant 30 R_{\odot}=7 \times 10^{-7} \mathrm{pc}$ is constant, then electron density $N_{e}(r) \propto r^{-2}$. If $N_{e}=10^{12} \mathrm{~cm}^{-3}$ at $r=30 R_{\odot}$ the hydrogen emission measure EM $=\int N_{e}(s) N_{\mathrm{H}}(s)$ ds may be computed along lines of sight which pass $\varrho$ pc from W stars. In the approximation that $\varrho$ is small compared with the distance of the star, $\mathrm{EM}=0.12 \pi \varrho^{-3} \mathrm{pc} \mathrm{cm}^{-6}$. For example, at $R=$ $=1200 \mathrm{pc}, \mathrm{EM}=1.9 \times 10^{6} \mathrm{pc} \mathrm{cm}^{-6}$ at $1^{\prime \prime}$ arc from the star, or $1.9 \times 10^{3} \mathrm{pc} \mathrm{cm}^{-6}$ at $10^{\prime \prime}$ arc. Nebulae for which $\mathrm{EM} \geqslant 400 \mathrm{pc} \mathrm{cm}^{-6}$ are commonly visible on $\mathrm{H} \alpha$ photographs. However, the star image would be competitive on ordinary photographs, and the hydrogen emission measure would not be appropriate for hydrogen-deficient $\mathbf{W}$ stars. According to Pengelly (1964) the intensity of recombination He II 6560 is 0.6 $\times$ the intensity of $\mathrm{H} \alpha$ in the limit of a low electron density and at $T_{e}=10^{4} \mathrm{~K}$. Likewise the intensity of $\mathrm{He}$ II 4686 is $4.4 \times$ the intensity of $\mathrm{H} \alpha$. We should probably find $N_{e}=2 N_{\mathrm{He}}{ }^{2+}$ rather than $N_{e}=N_{\mathrm{H}}{ }^{+}$in the envelopes of some W stars. Note also that, 
according to the adopted density law, $N_{e} \leqslant 10^{10} \mathrm{~cm}^{-3}$ at $r \geqslant 300 R_{\odot}$, and forbidden lines can be emitted. They may compete with $\mathrm{He}$ II lines in intensity as in planetary nebulae.

But one more question remains about the expanding envelope of a $\mathrm{W}$ star regarded as a special $\mathrm{H}$ II region. Is the Strömgren radius $r_{s}$ significantly larger than the star? Can the continuum photons of the core below $\lambda 912 \AA$ escape to photoionize the symmetric nebulae? If the envelope density $N_{e}=10^{12} \mathrm{~cm}^{-3}$ is constant inward from $r=30 R_{\odot}$ to the photosphere of a star of radius $r_{*}=6 R_{\odot}$ and effective temperature of $30000 \mathrm{~K}, r_{s}=29 N_{e}^{-2 / 3} \mathrm{pc}=2.9 \times 10^{-7} \mathrm{pc}=13 R_{\odot}$, as given by Spitzer (1968) for a standard 08 star. The actual radius $r_{s}$ will be larger if the $\mathrm{W}$-star core is larger or hotter or if $N_{e}$ is less. However, $N_{e}$ may be greater since the possible range is $10^{10}-10^{14}$ $\mathrm{cm}^{-3}$ (Underhill, 1968), and the density may follow the $r^{-2}$ law for constant-velocity ejection down to the photosphere. Doubly-ionized He regions are smaller than ionized $\mathbf{H}$ regions. Detailed models are in order, but an immediate conclusion is that some W envelopes may smother the stellar uv radiation, unless the envelopes are confined to the equatorial plane or some other non-isotropic configuration. We should be tempted to explain in this way the absence of symmetric nebulae or other nebulae around some W stars except for the complication that they may be doubles with companions that are independently able to photoionize surrounding nebulae.

Are W stars or their expanding envelopes detectable as radio sources? Davies et al. (1967) reported HD 16523 and HD 193793 at $2695 \mathrm{MHz}$ in a beam of $15^{\prime} \mathrm{HPBW}$. However, the former source is probably identifiable with 4C56.04 rather than the W star (Johnson, 1971), and no W star has been detected in a search with the Green Bank interferometer (Hjellming, 1971). Johnson (1971) looked at HD 9974, HD 168206, HD 177230, HD 187282, HD 190918, HD 191765, HD 193793, HD 211853 , and HD 214419 at $7795 \mathrm{HMz}$ in a beam of 4.4 HPBW. One source was found in the beam at HD 211853, but this is probably part of a (non-symmetric) nebula in the area. The answer to the question appears to be no, at present.

\section{Acknowledgements}

This report has been supported partially by the Lockheed Independent Research Program and partially by the NASA under contract NASW-1977. The OAO-2/WEP investigators have provided primary data under the guest-observer program. The Argentine Consejo Nacional de Investigaciones Científicas y Técnicas defrayed much of the cost of travel to the symposium. The hospitality of Dr J. Sahade and his colleagues in the Institudo de Astronomía y Física del Espacio is very much appreciated.

\section{References}

Bless, R. C.: 1971, private communication.

Bless, R. C. and Savage, B. D.: 1971, in A. D. Code (ed.), Symposium on Scientific Results from $O A O-2$, Amherst, p. 175.

Bradley, P. T. and Morton, D. C.: 1969, Astrophys. J. 156, 687. 
Carruthers, G. R.: 1968, Astrophys. J. 151, 269.

Code, A. D., Houck, T. E., McNall, J. F., Bless, R. C., and Lillie, C. F.: 1970, Astrophys. J. $161,377$. Courtès, G.: 1960, Ann. Astrophys. 23, 115 (Table IX).

Davies, J. G., Ferriday, R. J., Haslam, C. G. T., Moran, M., and Thomasson, P.: 1967, Monthly Notices Roy. Astron. Soc. $135,139$.

Davis, R. J.: 1971, Preliminary Catalog of Celescope Ultraviolet Observations, Smithsonian Institution, Ap. Obs., Cambridge.

Georgelin, Y. P. and Georgelin, Y. M.: 1970, Astron. Astrophys. 6, 349.

Georgelin, Y. P. and Monnet, G.: 1970, Astrophys. Letters 5, 239.

Hjellming, R. M.: 1971, private communication.

Houck, T. E.: 1971, in A. D. Code (ed.), Symposium on Scientific Results from O AO-2, Amherst, p. 479.

Johnson, H. M.: 1971, Astrophys. J: 167, 491.

Johnson, H. M. and Hogg, D. E.: 1965, Astrophys. J. 142, 1033.

Lozinskaya, T. A.: 1970, Astron. Zh. 47, 122.

Lozinskaya, T. A. and Esipov, V. F.: 1968, Astron. Zh. 45, 1153.

McGree, R. X. and Murray, J. D.: 1961, Australian J. Phys. 14, 260.

Minkowski, R.: 1965, in A. Blaauw and M. Schmidt (eds.), Galactic Structure, Univ. of Chicago Press, Chicago and London, p. 337.

Morton, D. C.: 1967, Astrophys. J. 147, 1017.

Morton, D. C.: 1970, Astrophys. J. 160, 215.

Parker, R. A. R.: 1963, Thesis, California Inst. of Technology.

Parker, R. A. R.: 1964, Astrophys. J. 139, 493.

Pengelly, R. M.: 1964, Monthly Notices Roy. Astron. Soc. 127, 145.

Pikelner, S. V.: 1959, Physics of the Interstellar Medium, Acad. Sci. U.S.S.R., Moscow, Figure 30.

Spitzer, Jr., L.: 1968, Diffuse Matter in Space, Interscience Pub., New York, Table 4.5.

Smith, A. M.: 1967, Astrophys, J. 147, 158.

Smith, L. F.: 1967, Astron. J. 72, 829.

Smith, L. F.: 1968a, in K. B. Gebbie and R. N. Thomas (eds.), Wolf-Rayet Stars, U.S. Government Printing Office, Washington, D.C., p. 21.

Smith, L. F.: 1968b, Monthly Notices Roy. Astron. Soc. 141, 317.

Smith, L. F.: 1971, in this Symposium; and in A. D. Code (ed.), Symposium on Scientific Results from OAO-2, Amherst, p. 429.

Smith, L. F. and Kuhi, L. V.: 1970, Astrophys. J. 162, 535.

Smith, L. F. and Batchelor, R. A.: 1970, Australian J. Phys. 23, 203.

Stecher, T. P.: 1968, in K. B. Gebbie and R. N. Thomas (eds.), Wolf-Rayet Stars, U.S. Government Printing Office, Washington, D.C., p. 65.

Stecher, T. P. and Milligan, J. E.: 1962, Astrophys. J. 136. 1.

Terzian, Y.: 1970, Astron. J. 75, 1155.

Underhill, A. B.: 1968, in M. Hack (ed.), Mass Loss from Stars, D. Reidel Pub. Co., DordrechtHolland, p. 17.

West, D. K.: 1971, in A. D. Code (ed.), Symposium on Scientific Results from OAO-2, Amherst, p. 441.

\section{DISCUSSION}

Underhill: Regarding the $O A O$ scans, what is the total filter bandpass at half intensity?

Johnson: Well, they are different from band to band, but they are roughly $200-400 \AA$. The passbands are such that they practically overlap at all places along the spectra. In other words, if there is any monochromatic line in the spectrum, it will be captured by one or more bands. So that it is true that the lines do affect the spectrum, but I do not think that we can say how from these data. In the case of HD 50896,1 found no suggestion of the presence of any very strong lines in the ultraviolet. But then may be I am wrong. I am really giving this report to show you the lack of information rather than give you much more information.

Morton: One problem in trying to estimate the interstellar contribution is that the interstellar $\mathrm{L} \alpha$ line, in many hot stars, is much weaker than one would expect from the $21 \mathrm{~cm}$ data. HD 50896 is close to Orion where we found at least a factor 5 less neutral hydrogen than we might expect from the 
$21 \mathrm{~cm}$ data. If that is the case, we would conclude that the contribution from the emission lines would be less.

Thomas: In the case of NGC 2359, how can one say that one has a nice spherical nebula when one sees a fountain-like ejection coming out from the star. What is your picture?

Johnson: In all of these nebular problems one is never able to get the perfect symmetry that one might have in a stellar problem. Consequently, when I say this is a nice shell around the star I am taking a little artistic license here and of course we have no direct evidence that this star actually is sitting inside a loop or a shell of nebula. It could be a foreground or background object but the coincidence looks a little too inviting to pass it by. Now it could be that this is not a shell but rather a spiral, but I call it a shell for convenience.

Thomas: I ask, whether it is conceivable that we do not have spherical symmetry, but may be something localized over certain points in the ejection.

Johnson: As a nebular astronomer I would like to call NGC 6888 'a neat shell' because in comparison to most nebulae, it is a neat shell. It is an elliptical feature perhaps lacking part of its perimeter, but nonetheless almost complete, and in stronger exposures, it is complete though weak on the one side. I draw your attention to the possible appearance of a general darkening of the inside of the shell, but outside we do not see that nebulosity. Now, as to the mechanism of ejection, of course we assume spherical symmetry. Certainly, stellar astronomers cannot blame us too much for that; it is the natural thing to do and we did believe that the star in the center does eject matter equally in all directions. We have not tried to explain the ellipticity or the finer points of departure from a spherical shell. For example, one might believe that a magnetic field did not permit the stuff to expand quite equally in all directions at the same speed. However, that is a relatively secondary feature of the argument, I think, at the present time.

Thomas: Could you not if you would want to be speculative and accept your model? In essence it says that mass goes out rapidly until it has swept up an interstellar medium mass equal to the ejected mass and then the velocity drops rapidly and reaches a small, asymptotic value where $4 \pi R^{2}{ }_{*} m_{*} v_{\text {eject }}=$ $=4 \pi R_{\text {neb }}{ }^{2} m_{\text {IsM }} v_{\text {neb }}$. This gives a spherical nebula, for uniform interstellar medium. But suppose you do not require such uniformity, could you not use the observed ellipticity of the nebula to infer a non-uniform distribution in the interstellar medium around the star, or is that too speculative? It can be made quantitative by exactly your approach.

Johnson: I think that is a pretty good speculation, because if the density of the ambient medium agrees with this correction you can explain both why the star is closest to one edge and why the stuff gets brighter where it does because the distance of travel is smaller and it piles up faster. So I do not object to that kind of an interpretation at all. I suppose in fact that the ambient medium outside the star may be able to control the shape of the shell whether it be a magnetic field or inhomogeneities of the ambient medium or whatever. The star can still be expelling the matter isotropically.

Smith: Just in support of what Johnson has just said, let me refer to NGC 3199 (see Proceedings 1968 WR Symposium, p. 41). It is a very nice example of the situation where the brightest parts of the nebula are closest to the star and faint extensions on the opposite side are at much greater distance. So I would support Johnson in the interpretation that the shape depends on the distribution of the interstellar medium. Let me summarise the general situation of rings. In 1968 there were 6 known, all associated with WN5, WN6 or WN8 stars, plus NGC 7635 with its Of star.

There is one recent development. Crampton has suggested the addition of two objects to the list of ring nebulae. One is the WN6 star HD 191765 which falls nicely into the previous generalization about the nebulae. However, his second one is a WN7 star which breaks the pattern. Neither are as well defined rings as the previous six and their reality needs confirmation. For the moment it looks like the association of ring nebulae with single WN5, WN6 and WN8 stars stands.

Paczyniski: It is really very nice to know that ring nebulae are observed around single stars only. I am surprised, though, that all these are WN stars, and that there is no WC star within ring nebulae, while there are single WC stars, I believe. As far as I know there is no systematic difference in the presently observed mass loss from single and binary WR stars. The outflow velocity is typically about $1000 \mathrm{~km}$ per second, considerably more then the velocity of orbital motion. Therefore, the mass outflow that is observed now is not seriously affected by the duplicity of some WR stars. It looks like ring nebulae were not created by the observed mode of mass loss, as in such case we should expect to see those nebulae around binary WR stars too. It seems that we should consider the possibility of a large mass loss that occurred in the past in single WR stars only. We know that there was a large mass loss in the past in the binaries: almost entire hydrogen-rich envelopes were transferred from the 
progenitors of present day WR components to their companions. It is possible that the progenitors of single WR stars have lost their massive hydrogen-rich envelopes in the past, and those envelopes are seen now as ring nebulae.

Thomas: Having been supported quickly by both Paczynski and Lindsey Smith on this idea of differential distribution of the interstellar medium, I hate to start backing down from it. But suppose you accept that every time $I$ have a ring like this, it simply reflects the interstellar medium density distribution. Then, I have, it seems to me, an enormous variation in the interstellar medium around individual stars. Is that something that one can accept happily? But suppose, just for fun, you accept it, then what?

Paczyński: But all those stars are believed to be single? I think there should be no relation between the distribution of interstellar matter and the duplicity of WR stars.

Thomas: Are you sure? If you think back on the question of how the stars evolved, of how they came together, what determines the distribution of the material in the interstellar medium around the star? You see, you get into all sorts of really speculation here, but if you want to start some place, you might as well be wild.

Johnson: I take it from Paczynski's remarks that he has given up the idea that the secondary star influences in an important way the loss of mass from the Wolf-Rayet star.

Paczyniski: No.

Johnson: You think the secondary star can capture a good part of the mass?

Paczyniski: There are two different things. One is the mass exchange in a close binary which is due to the fact that one of the components is so large that mass can freely flow to the companion. This does not require any ejection velocities, but if the ejection velocities are considerably larger than the velocity of the orbital motion, then the presence of the companion will produce a perturbation.

Johnson: A small perturbation?

Paczyniski: It depends upon the ratio of the expansion velocity to the velocity of the orbital motion.

Conti: Is there any expansion velocity that is in fact less than the orbital motion? For example, the orbital motion in V444 Cyg is small as the period is about 4 days. That is a lower limit to the expansion velocity, so it seems that the expansion velocity is always larger than the orbital velocity.

Paczynski: In that case the mass loss from the Wolf-Rayet stars, which is observed by means of violet displaced absorption lines, should hardly be affected by the presence of the companion. Of course it does not mean that the star which is observed to have a Wolf-Rayet spectrum has not lost a large amount of mass to the companion some time in the past. Without that mass exchange in the past one cannot explain the observed mass ratios and the observed over-luminosities. We have here two different kinds of mass loss processes.

Johnson: While we were walking to lunch, Paczynski very kindly explained to me how he thought planetary nebulae nuclei, some of which have WR-type spectra, came from Mira stars, and I wonder if he has any analogous process involving something other than a Mira star to explain the origin of population I WR stars? In other words, do you have any idea of what kind of star goes into a single Wolf-Rayet star?

Paczyniski: Well, there is a very recent suggestion due to Bisnovaty, Kogan and Nadyozhin, that a massive star cannot become a red supergiant because of mass outflow during the preceding evolutionary phases. Unfortunately, their calculations are not very precise. Still it is possible that the observed deficiency. of very luminous red supergiants may be due to a severe mass loss from these massive stars. In fact the mass loss from supergiants is observed, and the rate is the highest for the most luminous objects. Only a single star may develop an extended envelope as it has no companion that could capture the envelope too soon. Perhaps such an extended envelope is lost to the interstellar space and can be observed as a ring nebula. This is just a speculation.

Johnson: If the nebular shells, such as Hogg and I observed around some WR stars, are left over from slow mass loss during an earlier stage of the evolution of a single supergiant, Hogg and I are wrong to use the current $1000 \mathrm{~km} \mathrm{~s}^{-1}$ mass loss of WR stars to explain the nebular shells.

Underhill: That is right. It also ruins the age estimates of the star.

Johnson and Underhill: It takes away quite a few things from previous arguments.

Paczyniski: I believe the assumption of large previous mass loss is the easiest explanation for the fact that all the ring nebulae are observed around single stars only.

Johnson: I agree with you.

Thomas: It depends upon the phase of evolution. Suppose you argue that Wolf-Rayet stars occur early in the phase of evolution, then it would be in terms of the contraction hypothesis that we are 
fixing the surrounding medium. If it were late, which I gather that most of you believe, then maybe what most of you say is fine. But then, you talk about the red giants and there the velocities are low, so low that you cannot assume that the matter has really escaped unless you have quasi-slow previous ejections. Then the bigger ejection later catches up with it.

Paczyński: Yes.

Conti: But may I remind us all that we have Of stars and very luminous $O B$ supergiants in which the rocket UV observations themselves indicate very rapid mass loss. One might think that before a star is a red supergiant it is a blue supergiant and while it is a blue supergiant it is losing mass, lots of it, at a very rapid rate. The star is first a blue supergiant, then a red supergiant and when it loses all its envelope, you are down to the helium core and you have a Wolf-Rayet star.

Underhill: I would like to know what is the linear size of one of those rings, relative to Strömgren spheres. Now, if you have an $\mathrm{OS}$ star with an effective temperature of 40 to 50 thousand degrees, the simple theory gives, if I recall correctly, a Strömgren sphere of 20 parsecs. Everything within 20 parsecs of the star will be completely ionized so you will not see a hydrogen recombination spectrum which I presume is making your nebula visible. Therefore, we must assume that these nebulae are recombining and giving you $\mathrm{H} \alpha$ and that is the end of their Strömgren spheres.

Johnson: Around NGC 6888, if the distance is 1200 parsecs, it is about 2.6 parsecs in radius. It is quite a small $\mathrm{H}$ II region but probably the gas in the shell is somewhat denser than the gas in a typical $\mathrm{H}$ II region of ambient interstellar matter, so it is able to absorb the photons a little better.

Underhill: So, the shells are smaller and to make them visible you have to deduce a bigger density.

Smith: The densities, in several cases, were determined from the intensity ratio of $O$ II at $3727 \AA$.

Thomas: Do I understand that there are no WC stars that illuminate shells?

Smith: That is right, no population I WC stars have ring nebulae.

Paczyniski: With the possible exception of a very controversial object, $\mathrm{BD}+30^{\circ} 3639$, which is sometimes classified as a planetary nebula and sometimes as a Wolf-Rayet star.

Thomas: If I really accept your analogy and exclude the case you have just mentioned, then the evolutionary life of a WR star can be described as follows: first, it is a WC star; then, it becomes a red giant and loses some mass; and then, it becomes a WN star; furthermore, I see the mass loss because of the mass that came out of the giant phase. I am just reasoning phenomenologically following your logic; I do not see shells because I do not have any prior mass loss which gets swept up. 